\title{
Left Paraduodenal Hernia: A Rare Complication following Laparoscopic Appendectomy
}

\author{
Mathew A. Kozman ${ }^{1}$ and Oliver M. Fisher ${ }^{1,2}$ \\ ${ }^{1}$ Department of General Surgery, St George Hospital, Kogarah, NSW, Australia \\ ${ }^{2}$ School of Medicine, University of Notre Dame, Sydney, NSW, Australia \\ Correspondence should be addressed to Mathew A. Kozman; makozman@gmail.com
}

Received 22 July 2017; Revised 14 November 2017; Accepted 19 November 2017; Published 6 December 2017

Academic Editor: Tahsin Colak

Copyright (c) 2017 Mathew A. Kozman and Oliver M. Fisher. This is an open access article distributed under the Creative Commons Attribution License, which permits unrestricted use, distribution, and reproduction in any medium, provided the original work is properly cited.

\begin{abstract}
Paraduodenal hernias are rare congenital internal hernias accounting for $<2 \%$ of intestinal obstruction. Left paraduodenal hernias (LPDHs) into the fossa of Landzert are the more common type and result from abnormal rotation of the midgut and failure of peritoneal fusion. Sequelae of these hernias usually occur spontaneously in the 4th or 5th decade of life and are more common in males and have a significant risk of incarceration and subsequent strangulation. We describe a case of a 15-year-old female who develops a LPDH following laparoscopic appendectomy, resulting in jejunal incarceration and subsequent small intestinal obstruction. The patient discussed is from an atypical demographic, being young and female. In addition, the precipitating event prompting incarceration of the hernia appears to be the application of pneumoperitoneum, placement in the Trendelenburg position, and manipulation of small intestine for the purpose of facilitating laparoscopic appendectomy. To our knowledge, this is the first reported case of LPDH exacerbated by laparoscopic procedure.
\end{abstract}

\section{Introduction}

Paraduodenal hernias are rare congenital internal hernias accounting for $<2 \%$ of intestinal obstruction, most commonly involving jejunum [1]. Left paraduodenal hernias (LPDHs) into the fossa of Landzert are the more common type and result from abnormal rotation of the midgut and failure of peritoneal fusion [2, 3]. Sequelae of these hernias usually occur spontaneously in the 4 th or 5 th decade of life and are more common in males $[2,4]$. Patients with LPDH have a significant risk of incarceration and subsequent strangulation, associated with high mortality rates [5]. We report on an atypical event of incarcerated LPDH.

\section{Case Report}

A 15-year-old female presented with a 12-hour history of gradual onset right iliac fossa abdominal pain associated with nausea, vomiting, anorexia, and fevers. She denied urinary symptoms. Her last menstrual period was 3 months prior and was an irregular occurrence. She had no similar previous episodes. She had no significant past medical or surgical history and took no regular medications.

On examination, she was alert, orientated, and appeared well. Vital signs were within normal limits, and she was afebrile. Abdominal examination revealed tenderness in the lower abdomen, worse in the right iliac fossa. Rovsing's sign was present.

Investigations revealed a normal urine analysis, and subsequent culture was negative. White blood cell count was $19.1 \times 109 / \mathrm{L}$ with neutrophilia. C-reactive protein was $3.0 \mathrm{mg} / \mathrm{L}$. Abdominal ultrasound showed a noncompressible, avascular, hypoechoic tubular structure with well-defined margins measuring $9.2 \mathrm{~mm}$, extending from the mid-pelvis to the left side. This was suspicious for appendicitis.

The patient underwent a diagnostic laparoscopy and appendectomy. This was performed using a $10 \mathrm{~mm}$ infraumbilical hasson port and two further $5 \mathrm{~mm}$ working ports. The patient was positioned supine with bed tilted head-down (Trendelenburg position) and rolled leftward. 
High-flow carbon dioxide insufflation of $12 \mathrm{mmHg}$ was used, and small intestine was manoeuvred out of the pelvis into the upper abdomen. Early acute appendicitis with no perforation was found, and laparoscopic appendectomy was performed. Histopathology confirmed acute appendicitis.

The following day, the patient complained of severe and worsening abdominal pain. She was unable to tolerate oral intake. On examination, she appeared to be in pain. Tachycardia of 120 beats per minute was noted; otherwise, vital signs were within normal limits. Her abdomen was distended and tender with signs of generalised peritonism. Blood tests were unremarkable, with reduced white cell count compared with initial presentation. Due to this clinical picture, no further imaging studies were arranged, and she was taken back to the operating theatre for exploratory laparoscopy.

Upon laparoscopy, dilated small intestinal loops and copious free fluid were found. Conversion to laparotomy was performed due to impeded laparoscopic view. This revealed a small bowel obstruction with transition point at the paraduodenal region. An LPDH diagnosed with a long segment of jejunum was seen herniating through a defect to the left of the fourth part of the duodenum and accumulating in the fossa of Landzert (Figures 1 and 2). Reduction of the hernia was performed, ensuring preservation of the inferior mesenteric vein and middle colic artery branches. No widening of the hernia neck was required to successfully reduce the hernia. An approximately $15 \mathrm{~cm}$ segment of jejunum displayed signs of ischaemia but was viable upon hernia reduction and warm pack wrapping. The peritoneal hernia sac was excised, and the paraduodenal hernia orifice was closed using 2-0 vicryl and the sac as a patch. The patient made an uneventful recovery and was discharged home 5 days later.

\section{Discussion}

Internal hernias result from protrusion of the viscera through an opening in the peritoneum or mesentery [6]. They account for $<2 \%$ of intestinal obstruction [1]. Paraduodenal hernias account for $30-50 \%$ of all internal hernias, which are also the most common type of congenital internal hernias [5]. Paraduodenal hernias into the left paraduodenal fossa of Landzert are three times more common than those into the right paraduodenal fossa of Waldayer [2]. The left paraduodenal fossa results from failure of mesenteric fusion with the parietal peritoneum and malrotation of the midgut resulting in development of a potential space [3]. LPDH occurs when small intestine prolapses posteroinferiorly into this fossa (of Landzert), which is bounded by the fourth part of the duodenum, the posterior peritoneum, the inferior mesenteric vein, and left branches of the middle colic artery $[7,8]$. This may result in small bowel incarceration, obstruction, and subsequent ischaemia.

Patients most commonly present in the 4 th to 5 th decades or life [2], and there is a $3: 1$ male preponderance [4]. Presentation is variable depending on the severity of the hernia sequelae and the presence of obstruction [2]. Approximately $50 \%$ of patients recall previous recurring abdominal pain of nonspecific nature [9]. As such, the entity poses a diagnostic challenge, with majority of cases identified only at operation

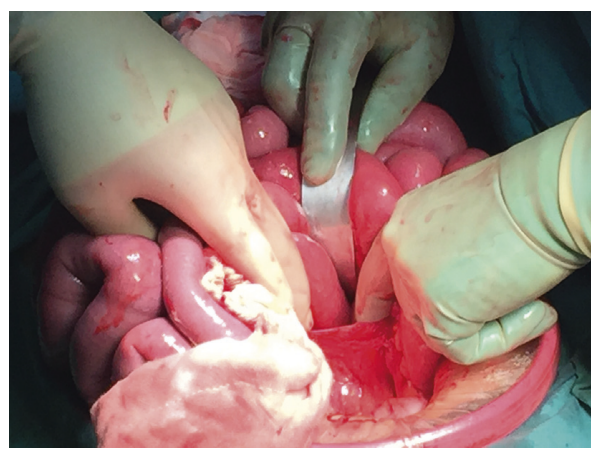

Figure 1: Intraoperative photograph showing left paraduodenal hernia: left paraduodenal hernia (LPDH) occurring when small intestine prolapses posteroinferiorly into this fossa (of Landzert), which is bounded by the fourth part of the duodenum, the posterior peritoneum, the inferior mesenteric vein, and left branches of the middle colic artery. LPDH being retracted in order to facilitate reduction of the hernial contents, namely, small intestine.

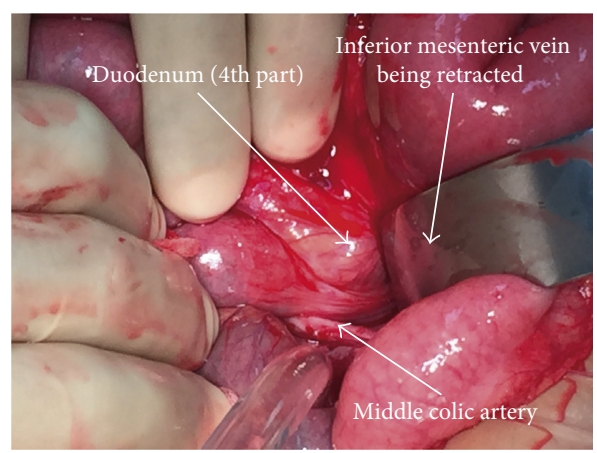

FIGURE 2: Intraoperative photograph showing left paraduodenal hernia: left paraduodenal hernia (LPDH) occurring when small intestine prolapses posteroinferiorly into this fossa (of Landzert), which is bounded by the fourth part of the duodenum, the posterior peritoneum, the inferior mesenteric vein, and left branches of the middle colic artery. LPDH borders displayed.

[10]. Patients with LPDH have a 50\% lifetime risk of hernia incarceration with $20-50 \%$ mortality for acute presentations; hence, operative management is recommended regardless of symptoms [5].

The most effective preoperative diagnostic tool is the computed tomography (CT) scan [11]. This may reveal a cluster of small bowels at the ligament of Treitz with or without associated findings of small intestine obstruction [2]. An additional compression on the posterior stomach and distal duodenum results in inferior displacement of transverse colon and shifting of the mesenteric truck to the right [12]. However, instances of acute abdomen warrant omission of preoperative imaging and immediate exploratory laparotomy.

Treatment methods reported in the literature include laparoscopy or laparotomy and repair. However, exploratory laparotomy is more often reported, especially in the setting of an acute complication such as strangulation, perforation, 
or large distension from obstruction [13]. In addition, laparotomy may be more appropriate in circumstances where laparoscopy may not be possible or dangerous such as significant adhesions, haemodynamic instability, and contraindication to pneumoperitoneum [13]. Nonetheless, the laparoscopic approach to management of this condition has become increasingly prevalent in the literature [14]. Regardless of the approach, basic principles of hernia repair are adopted, namely, reduction of hernia contents and repair of hernia defect [9]. Excision of the hernia sac has been described but is not mandatory given the potential for injury to the colic vessels [14]. Correct identification and preservation of the vascular structures that constitute the hernia neck is essential [12]. Rarely, widening of the hernia neck is required to reduce the contents and may involve incision of constricting peritoneal fold inferiorly and even division of the inferior mesenteric vein in more difficult cases [15].

A literature search of PubMed back to 1980 discovered only approximately 50 reported cases of intestinal obstruction secondary to a LPDH. However, to our knowledge, this is the first reported case of LPDH exacerbated by laparoscopic pneumoperitoneum and intraoperative patient positioning. This case is particularly interesting for a few reasons. Firstly, the patient demographic is not typical of this disease. Our patient was a young female, while reported cases more commonly describe males in the 4 th to 5 th decades of life. Secondly, the patient described no symptoms at any time prior to the day of her appendectomy. Although the patient clearly possessed the congenital anomaly of a left paraduodenal fossa, the application of pneumoperitoneum, placement in the Trendelenburg position, and manipulation of small intestine for the purpose of facilitating laparoscopic appendectomy appear to have precipitated the LPDH and subsequent incarceration. In this instance, the diagnosis was not made prior to laparotomy as in most cases presenting with an acute abdomen. Although diagnostic laparoscopy was performed upon return to theatre, gross small bowel distension from obstruction prevented further progression via the laparoscopic approach.

\section{Consent}

Written informed consent was obtained from the patient for publication of this case report and accompanying images.

\section{Conflicts of Interest}

The authors declare that there are no conflicts of interest regarding the publication of this article.

\section{Authors' Contributions}

Mathew A. Kozman was the operating surgeon for the appendectomy and assistant surgeon for the laparotomy. $\mathrm{He}$ wrote the manuscript and conceived and designed the study. Mathew A. Kozman and Oliver M. Fisher were responsible for drafting and revising the article content and for the final approval of the manuscript prior to submission.

\section{References}

[1] S. Akbulut, "Unusual cause of intestinal obstruction: left paraduodenal hernia," Case Reports in Medicine, vol. 2012, Article ID 529246, 2 pages, 2012.

[2] R. Downes and S. O. Cawich, "A case of a paraduodenal hernia," International Journal of Surgery Case Reports, vol. 1, no. 2, pp. 19-21, 2010.

[3] A. Husain, S. Bhat, A. K. Roy et al., "Internal hernia through paraduodenal recess with acute intestinal obstruction: a case report," Indian Journal of Surgery, vol. 74, no. 4, pp. 354-355, 2012.

[4] R. Manji and G. L. Warnock, "Left paraduodenal hernia: an unusual cause of small-bowel obstruction," Canadian Journal of Surgery, vol. 44, no. 6, pp. 455-457, 2001

[5] W. Al-Khyatt, S. Aggarwal, J. Birchall, and T. E. Rowlands, "Acute intestinal obstruction secondary to left paraduodenal hernia: a case report and literature review," World Journal of Emergency Surgery, vol. 8, no. 1, p. 5, 2013.

[6] L. C. Martin, E. M. Merkle, and W. M. Thompson, "Review of internal hernias: radiographic and clinical findings," American Journal of Roentgenology (AJR), vol. 186, no. 3, pp. 703-717, 2006.

[7] O. Armstrong, A. Hamel, B. Grignon et al., "Internal hernias: anatomical basis and clinical relevance," Surgical and $R a$ diologic Anatomy, vol. 29, no. 4, pp. 333-337, 2007.

[8] P. Zonca, T. Maly, D. J. Mole, and J. Stigler, "Treitz's hernia," Hernia, vol. 12, no. 5, pp. 531-534, 2008

[9] J. R. Gusz and L. M. Wright, "Intestinal obstruction secondary to left paraduodenal hernia," Journal of Surgical Case Reports, vol. 2015, no. 7, pp. 1-3, 2015.

[10] L. Barbosa, A. Ferreira, A. A. Povoa, and J. P. Maciel, "Left paraduodenal hernia: a rare cause of small bowel obstruction in the elderly," BMJ Case Reports, vol. 2016, pp. 1-3, 2016.

[11] A. Blachar, M. P. Federle, and S. F. Dodson, "Internal hernia: clinical and imaging findings in 17 patients with emphasis on CT criteria," Radiology, vol. 218, no. 1, pp. 68-74, 2001.

[12] D. Kabbani, A. Salem, and D. K. Holloway, "Paraduodenal herniation: an internal herniation in a virgin abdomen," International Journal of Surgery Case Reports, vol. 5, no. 12, pp. 1148-1150, 2014.

[13] B. P. Parmar and R. S. Parmar, "Laparoscopic management of left paraduodenal hernia," Journal of Minimal Access Surgery, vol. 6, no. 4, pp. 122-124, 2010.

[14] M. Zizzo, N. Smerieri, I. Barbieri et al., "Laparoscopic treatment of acute small bowel obstruction due to left paraduodenal hernia: a case report and literature review," International Journal of Surgery Case Reports, vol. 20, pp. 87-91, 2016.

[15] G. V. Kulkarni, H. P. Salgaonkar, P. C. Sharma et al., "Laparoscopic repair of left paraduodenal hernia: report of two cases and review of the literature," Asian Journal of Endoscopic Surgery, vol. 9, no. 2, pp. 157-160, 2016. 


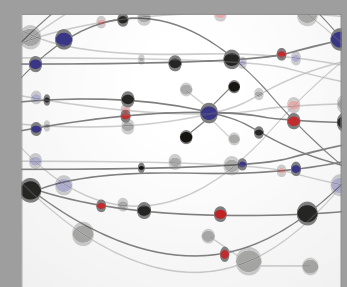

The Scientific World Journal
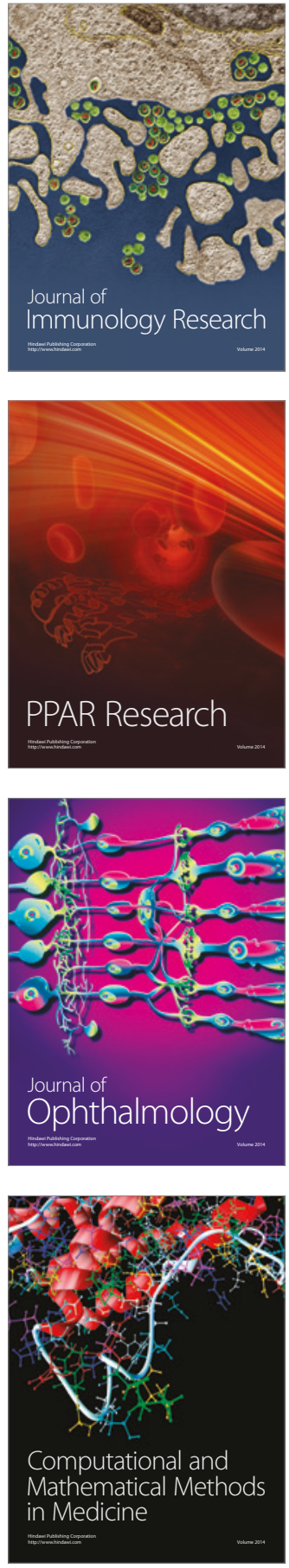

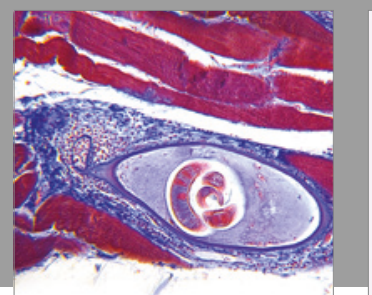

Gastroenterology Research and Practice
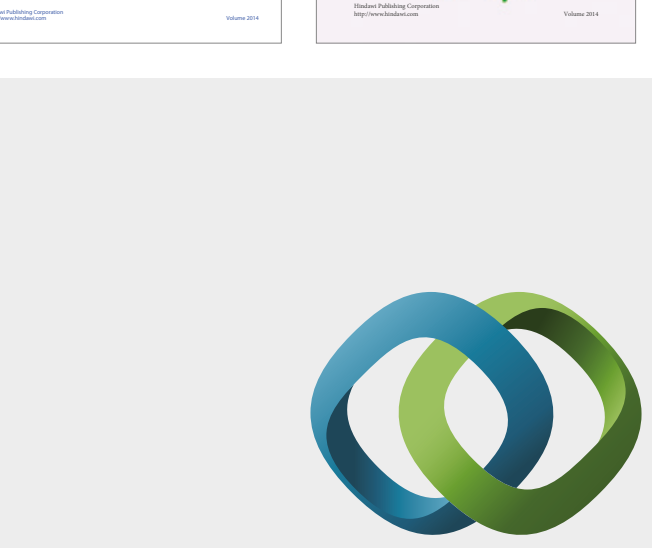

\section{Hindawi}

Submit your manuscripts at

https://www.hindawi.com
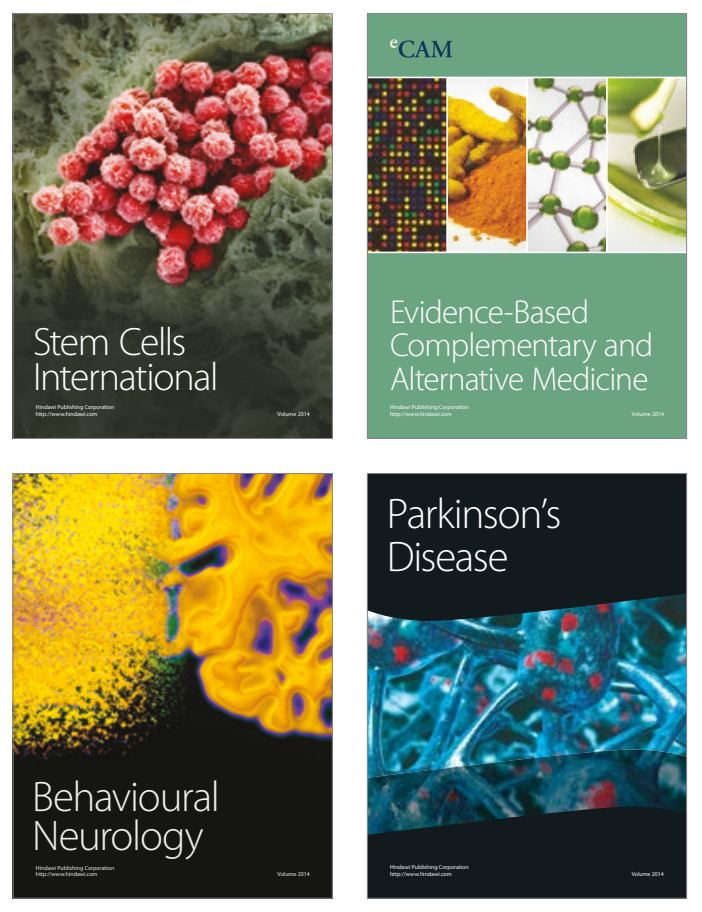
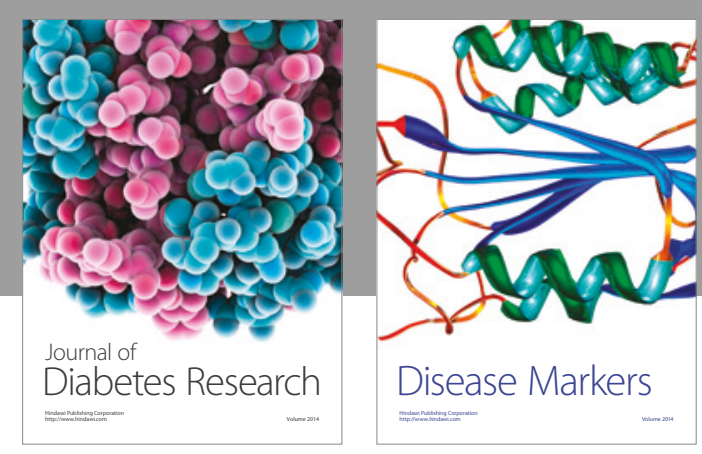

Disease Markers
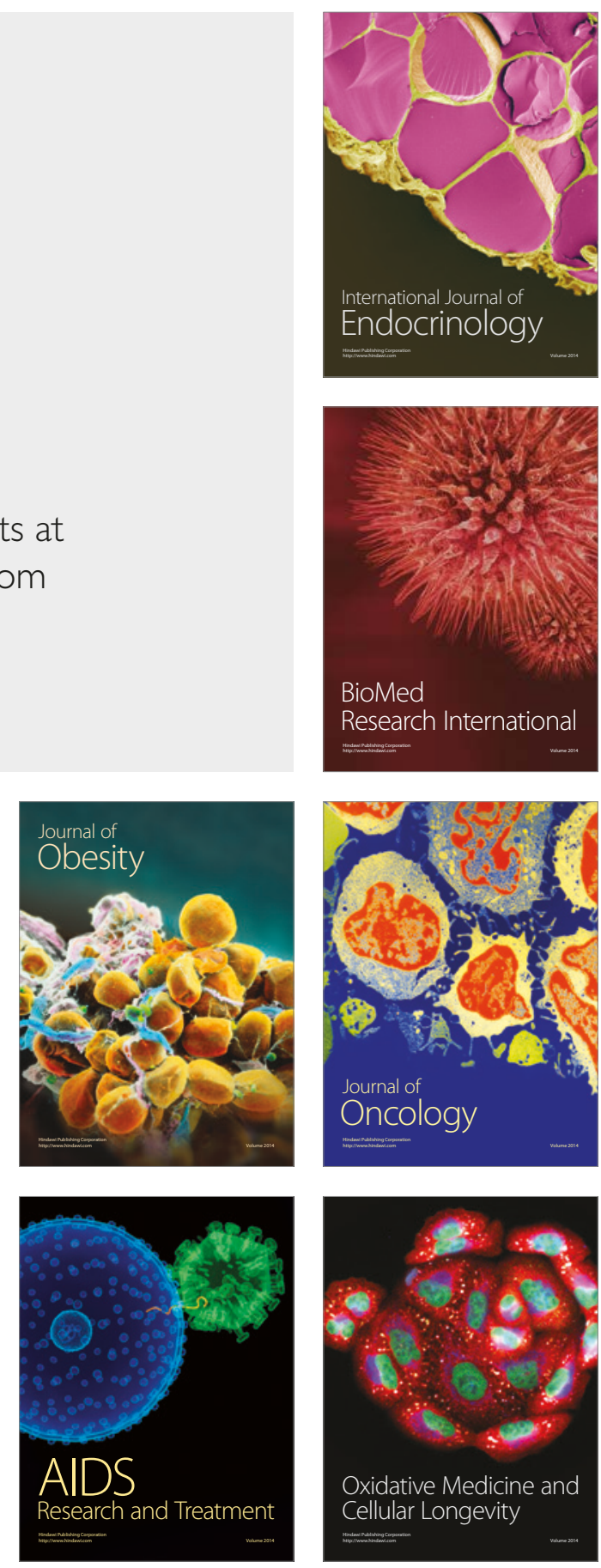